\title{
Control de inventario y rentabilidad en una empresa ferretera de Manantay - Perú
}

Inventory control and profitability in a hardware company in Manantay - Peru

Controle de estoque e lucratividade em uma empresa de hardware em Manantay - Peru

Nieves del Pilar Pizzán-Tomanguillo

nievesdelpilarpizzan1993@gmail.com CNL ASESORES - Perú

ORCID: https://orcid.org/0000-0001-9933-8738

Carlos Daniel Rosales Bardalez

bardalez.daniel@gmail.com

Universidad Peruana Unión - Perú

ORCID: https://orcid.org/0000-0002-2311-1577

Ceron Shapiama Cris Charly cristina14119226@gmail.com

Universidad César Vallejo - Perú

ORCID: https://orcid.org/0000-0002-4341-5711

\section{RESUMEN}

El presente informe tuvo como objetivo general: Determinar el nivel de relación entre control de inventario y rentabilidad en la empresa "El mundo de los Repuestos JR E.I.R.L"., periodo 2018-2019. En cuanto a la estructura metodológica del estudio fueron: enfoque cuantitativo de tipo básico, nivel descriptivo correlacional, diseño no experimental de corte longitudinal, la muestra determinada por 48 estados financieros (24 estados de situación financiera y 24 estados de resultados) mensuales de la empresa, la técnica empleada fue el análisis documental. Se llegó a la conclusión que existe relación entre la rotación de inventario y la rentabilidad sobre activos, porque se constató un p-valor $=0.027<0.05$, así también, existe relación entre la rotación de inventario y la rentabilidad neta con un p-valor $=0.048<0.05$, por lo contrario, no existe relación entre la rotación de inventario y rentabilidad sobre el capital. Por consiguiente, existe relación entre plazo medio de existencia y la rentabilidad neta, porque se constató un p-valor $=0.00<0.05$, además existe relación entre plazo medio de existencia y rentabilidad sobre activo, porque se constató un p-valor $=0.005<0.05$, finalmente, existe relación entre plazo medio de existencia y la rentabilidad sobre el capital, porque se constató un p-valor $=0.008<0.05$.

Palabras clave: Índice de rotación de existencia, Plazo medio de existencia, Rentabilidad

\section{ABSTRACT}

The present investigation had as general objective: To determine the level of relationship between inventory control and profitability in the company "El mundo de los Repuestos JR E.I.R.L.", Period 2018-2019. Regarding the methodological structure of the study, they were: a basic quantitative approach, a correlational descriptive level, a non-experimental design with a longitudinal cut, a sample determined by 48 financial statements (24 financial statements and 24 income statements) monthly. of the company, the technique used was documentary analysis. It was concluded that there is a relationship between inventory turnover and return on assets, because a p-value $=0.027<0.05$ was found, as well as, there is a relationship between inventory turnover and net profitability, because a p-value $=0.048<0.05$, on the contrary, there is no relationship between inventory turnover and return on equity. Consequently, there is a relationship between average life time and net profitability, because a p-value $=0.00<0.05$ was found, and there is also a relationship between average life time and return on assets, because a p-value $=0.005<0.05$, finally, there is a relationship between the average term of existence and the return on capital, because in table 9 , a p-value $=0.008<0.05$ was found.

Keywords: Stock turnover index, Average life span, Profitability 


\section{RESUMO}

O objetivo geral deste relatório foi: Determinar o nível de relacionamento entre controle de estoque e lucratividade na empresa "El mundo de los Repuestos JR E.I.R.L.", período 2018-2019. Quanto à estrutura metodológica do estudo, foram elas: abordagem quantitativa do tipo básico, nível correlacional descritivo, desenho não experimental de corte longitudinal, amostra determinada por 48 demonstrações financeiras (24 demonstrações de posição financeira e 24 demonstrações de resultados) mensais de empresa, a técnica utilizada foi a análise documental. Concluiu-se que existe uma relação entre giro de estoque e retorno sobre os ativos, pois foi encontrado um valor de $\mathrm{p}=0,027<0,05$, assim como uma relação entre giro de estoque e lucratividade líquida com valor de $\mathrm{p}=0,048<0,05$, no pelo contrário, não há relação entre giro de estoque e retorno sobre capital. Portanto, existe uma relação entre o prazo médio de existência e a rentabilidade líquida, pois foi encontrado um valor de $\mathrm{p}=0,00<0,05$, além disso existe uma relação entre o prazo médio de existência e o retorno sobre os ativos, pois um valor de $\mathrm{p}=0,005<$ foi encontrado 0,05 , enfim, existe uma relação entre o prazo médio de existência e o retorno sobre o capital, pois foi encontrado um $\mathrm{p}$-valor $=0,008<0,05$.

Palavras-chave: Taxa de giro de estoque, Prazo médio de existência, Rentabilidade

\section{INTRODUCCIÓN}

El control de inventario es una de las actividades más complejas en las empresas, por cuanto implica mantener mercadería para protegerse contra incertidumbres. Esta situación se hace más aguda en economías emergentes, donde factores internos propios de la empresa y externo de tipo económico aquejan esta gestión y las decisiones que se toman con base a las aplicaciones de las políticas y lineamiento desarrolladas para tal fin.

La gestión de inventario involucra optimizar tiempo y lugar, la reducción de espacios genera efectos positivos en la rotación de las existencias, sabiendo que la mala ubicación genera retrasos en la comercialización de los productos, por ello es importante disponer de un almacén ordenado que minimice el tiempo de distribución, visto que genera un incremento en la rentabilidad. Asimismo, es importante que los responsables del control efectúen un análisis y seguimiento de las necesidades y demandas existes, para un alto nivel de rotación del producto (Peña y Silva, 2016).

La economía peruana está en incesante crecimiento y la industria automotriz es un sector económico importante en el mundo por ingresos que este genera, en tal sentido es importante el empleo de lineamiento y normas que encaminen a la rotación continua de los productos. Sin embargo Contreras et al. (2018), sostienen que dichas empresas presentan problemas continuos en la rotación de sus existencia, la misma que se debe a la falta de procedimiento y políticas que se ajusten a la realidad de la empresa a la falta de empleo de los registros y papeles de control que demuestren un seguimiento continuo de la calidad y cantidad de los productos; por lo tanto, Manosalvas et al. (2020) señalan que el inventario en dichas empresas deben ser almacenados, contados y manejados eficazmente, a fin de reducir costos y evitar pérdidas por lo demás es indispensable que mantengan su inventario en mejora continua para asegurar un buen resultado en beneficio de sus clientes y las finanzas del negocio.

Las realidades expresas, no son ajenas a lo suscitado en la empresa "El mundo de los Repuestos JR empresa jurídica constituido como EIRL con RUC. 20600421434, dedicada a la venta de partes, piezas y accesorios de motocicletas. De la revisión a los lineamentos del control de inventarios otorgado por gerencia y la verificación de la documentación que respalda las operaciones realizadas, se identificaron hechos incorrectos que dificultan el control de los inventarios.

Entre los hechos más relevantes, es la inadecuada verificación de la calidad y cantidad de los productos recepcionados, asimismo gerencia dio a conocer que en un caso ellos solicitaron 305 unidades de llantas. Sin embargo, se recepcionaron 303 unidades del mismo modo ocurre con las pinturas donde solo ingresaron 153 de 160 unidades. 
Asimismo, se constató que los productos (piezas, pinturas y accesorios) almacenados se deterioran muestra de ello se refleja en las cadenas, kilometrajes, cuyos accesorios se oxidan o rompen. La situación descrita se debe a la inadecuada ubicación de los productos que permite la pronta perdida de la calidad de los productos. Seguidamente se observó que gerencia no implementa políticas que encaminen el buen almacenamiento, es decir la organización de las mercaderías por línea de comercialización en consecuencia esta situación descrita produce retrasos en la venta de los productos y atención a los clientes.

En el marco brevemente descrito, se formula el siguiente problema general: ¿Cuál es el nivel relación entre control de inventario y rentabilidad en la empresa "El mundo de los Repuestos JR E.I.R.L.”, Distrito de Manantay, periodo 2018-2019? y seguidamente los problemas específicos: ¿Cuál es el nivel de relación entre rotación de inventario y rentabilidad sobre activos en la empresa "El mundo de los Repuestos JR E.I.R.L.”, Distrito de Manantay, periodo 2018-2019?, ¿Cuál es el nivel de relación entre rotación de inventario y rentabilidad neta en la empresa "El mundo de los Repuestos JR E.I.R.L.”, Distrito de Manantay, periodo 2018-2019?; ¿Cuál es el nivel de relación entre rotación de inventario y rentabilidad sobre el capital en la empresa "El mundo de los Repuestos JR E.I.R.L.", Distrito de Manantay, periodo 2018-2019?; ¿Cuál es el nivel de relación entre plazo medio de existencias y rentabilidad sobre activos en la empresa "El mundo de los Repuestos JR E.I.R.L.", Distrito de Manantay, periodo 2018-2019?; ¿Cuál es el nivel de relación entre plazo medio de existencias y rentabilidad neta en la empresa "El mundo de los Repuestos JR E.I.R.L.", Distrito de Manantay, periodo 2018-2019?; ¿Cuál es el nivel de relación entre plazo medio de existencias y rentabilidad sobre el capital en la empresa "El mundo de los Repuestos JR E.I.R.L.", Distrito de Manantay, periodo 2018-2019?

El objetivo general que persigue la investigación es: Determinar el nivel de relación entre control de inventario y rentabilidad en la empresa "El mundo de los Repuestos JR E.I.R.L.", Distrito de Manantay, periodo 2018-2019., los específicos: Determinar el nivel de relación entre rotación de inventario y rentabilidad sobre activos en la empresa "El mundo de los Repuestos JR E.I.R.L.", Distrito de Manantay, periodo 2018-2019; Determinar el nivel de relación entre rotación de inventario y rentabilidad neta en la empresa "El mundo de los Repuestos JR E.I.R.L.", Distrito de Manantay, periodo 2018-2019; Determinar el nivel de relación entre rotación de inventario y rentabilidad sobre el capital en la empresa "El mundo de los Repuestos JR E.I.R.L.", Distrito de Manantay, periodo 20182019; Determinar el nivel de relación entre plazo medio de existencias y rentabilidad sobre activos en la empresa "El mundo de los Repuestos JR E.I.R.L.", Distrito de Manantay, periodo 2018-2019; Determinar el nivel de relación entre plazo medio de existencias y rentabilidad neta en la empresa "El mundo de los Repuestos JR E.I.R.L.”, Distrito de Manantay, Periodo 2018-2019; y Determinar el nivel de relación entre plazo medio de existencias y rentabilidad sobre el capital en la empresa "El mundo de los Repuestos JR E.I.R.L.”, Distrito de Manantay, periodo 2018-2019.

Al mismo tiempo se presentan las justificaciones que comprenden las razones y motivos de la realización del estudio, bajo un concepto de implicancia práctica porque otorga a los responsables y gerente de la empresa información relevante y asertiva de los problemas relacionados al control de inventario, a fin de establecer alternativas de mejora y contribuir en la rentabilidad de esta. También se justifica de manera teórica, porque la revisión y el análisis de documentos permitirá crecentar el conocimiento del investigador en relación con las variables estudiadas, que le permitirá desarrollar los objetivos propuestos. Desde un concepto metodológico, se justifica puesto que se aplicaron técnicas y método de recolección de datos; asimismo se desarrollaron instrumentos y bibliografía que podrán ser empleados por estudiantes que presenten la misma problemática y por consiguiente la solución de los objetivos planteados en las investigaciones. 
La hipótesis general que responde al problema es: Existe relación entre control de inventarios y rentabilidad en la empresa "El mundo de los Repuestos JR E.I.R.L.", Distrito de Manantay, periodo 2018-2019, seguidamente los específicos: Existe relación entre rotación de inventario y rentabilidad sobre activos en la empresa "El mundo de los Repuestos JR E.I.R.L.", Distrito de Manantay, periodo 2018-2019. Existe relación entre rotación de inventario y rentabilidad neta en la empresa "El mundo de los Repuestos JR E.I.R.L.”, Distrito de Manantay, periodo 2018-2019. Existe relación entre rotación de inventario y rentabilidad sobre el capital en la empresa "El mundo de los Repuestos JR E.I.R.L.", Distrito de Manantay, periodo 2018-2019. Existe relación entre plazo medio de existencias y rentabilidad sobre activos en la empresa "El mundo de los Repuestos JR E.I.R.L.", Distrito de Manantay, periodo 2018-2019. Existe relación entre plazo medio de existencias y rentabilidad neta en la empresa "El mundo de los Repuestos JR E.I.R.L.", Distrito de Manantay, periodo 2018-2019. Y Existe relación entre plazo medio de existencias y rentabilidad sobre el capital en la empresa "El mundo de los Repuestos JR E.I.R.L.”, Distrito de Manantay, periodo 2018-2019.

En el presente informe se presenta en el apartado dos, el fundamento teórico, en la que se muestra las investigaciones relacionadas a las variables en estudio (control de inventario y rentabilidad), como también la base teórica y dimensiones, por otro lado en el apartado tres, se desarrolla los procedimientos metodológicos, donde se describe la metodología utilizada, las técnicas e instrumentos aplicados en la investigación, en el apartado cuatro, se desarrollan los resultados y discusiones, se presenta los resultados en forma narrativa y grafica de acuerdo a los objetivos planteados, se realiza un análisis crítico sobre las mismas, para posteriormente comparar los mismos con otros estudios similares, sigue las conclusiones, se presenta en forma resumida la respuesta al objetivo del estudio, como se legró a lo que se llegó, también mostrar las limitaciones y sugerir futuras investigaciones, por último se muestra las referencias utilizadas en el desarrollo del artículo.

\section{FUNDAMENTO TEÓRICO}

\subsection{Antecedentes}

A fin de brindar mayor consistencia a la investigación, se ha empleado investigaciones que sustentan el estudio desde un contexto internacional y nacional.

Desde un ámbito internacional, Asencio y González (2017) en su artículo sobre el inventario como determinante en la rentabilidad de las distribuidoras farmacéuticas, cuyo nivel fue correlacional y de enfoque cuantitativo donde su población y muestra estuvo constituida por 25 trabajadores de la organización JRC Pharma, concluye que las actividades del control de inventario son deficientes en un $42 \%$ y un $67 \%$ y afirmó que la compañía carece de un control de existencias; asimismo existe deficiente manejo en la adquisición de existencias en un 53\%, por tanto, la incidencia es positiva considerable de 0.891 de correlación de Pearson. Cabe mencionar, que el índice de rentabilidad del capital no muestra dependencia del control de inventario, visto que el p valor $=0.091>0.05$.

Jara y Sánchez (2017) en su investigación sobre el análisis para mejorar el manejo de inventarios de una comercializadora donde su el nivel fue descriptivo y enfoque cuantitativo y su población y muestra fue una empresa comercializadora de puebla, llegaron a las siguientes conclusiones: Para una organización manejar las existencias implica costos elevados; por ello, es importante mantener a tope los inventarios y no realizar exceso de adquisiciones. La organización dejo evidencia de faltantes de mercadería por falta de un control adecuado en el almacén.

Quinde y Ramos (2018) en su artículo sobre el efecto del control del inventario en la rentabilidad cuyo nivel de estudio fue causal y de enfoque cuantitativo cuyo población y muestra estuvo conformado por 4 colaboradores de la empresa Disensa Ramírez S.A., llegaron a la conclusión que la empresa tiene un proceso de venta favorable; asimismo, la rotación mensual del inventarios fue 
de 3.94 , el margen de utilidad fue un $26 \%$ y rendimiento sobre activos fue 0.86 , por lo tanto mostró que la rotación de inventario incide de forma positiva en la rentabilidad, con un p valor $=0.00<0.05$, y con un indicador de correlación de Pearson de 0.781.

Terán (2017) en su tesis sobre el efecto del control de los inventarios (NIC 2) en la rentabilidad de la empresa PROVEC INDUSTRIAL de la ciudad de Ambato, determinó que la organización tuvo una perdida por $\$ 35,660$ y un rendimiento del $8 \%$, además de encontrar deficiencias en el proceso de compras demostrando un efecto positivo entre el control de inventario y la rentabilidad, visto que el nivel de significancia fue $=0,01$, cuya correlación de Rho Spearman es de positiva de 0.745.

Desde un contexto nacional, López y Galarreta (2018) en su artículo sobre elementos del inventario para reducir los costos cuyo nivel del estudio fue causal y de enfoque cuantitativo donde la población y muestra fueron los datos del 2015 - 2016, concluyen que la elaboración de la clasificación ABC permitió repartir cada artículo de las existencias, asimismo, con la propuesta elaborada hubo una reducción de costos de inventarios de $\mathrm{S} / 38,102$ a $\mathrm{S} / 29,259$, lo cual representa un $23.21 \%$ de manera correspondiente. Además, existe una relación positiva entre el inventario y la reducción de costo, con un índice de correlación de Pearson de 0.689. Por consiguiente, la rotación del inventario en días presenta un límite de 12 días al mes, por lo que ha tenido un efecto positivo en las ventas S/ 56,521.00 y diversificación de los productos en el periodo 2019,

Ángulo (2019) en su investigación sobre la relación del control interno en la gestión de inventarios de la empresa constructora Peter Contratistas S.R. Ltda. Donde el nivel de estudio fue correlacional y enfoque cuantitativo cuya técnica empleada fue la encuesta, concluye que el $88.9 \%$ manifestó que el control interno se realizaba de forma adecuada, del mismo modo el $94.4 \%$ refirió que la gestión de existencias es eficiente en lo que demostró que el control interno se vincula de forma favorable en la gestión de existencias con un valor de chi cuadrado $(x 2=4,656$ con $\mathrm{p}=0,000)$

Segura (2019) en su investigación sobre el análisis de las transacciones de la rentabilidad de nivel de estudio fue descriptivo y enfoque cualitativo donde su población y muestra estuvieron conformados por los estados financieros del 2016, 2017 y 2018, concluye que la rentabilidad del activo fue $1.02 \%,-2.64 \%$ y $1.69 \%$. y respecto a la rentabilidad sobre el patrimonio fue, $7.41 \%,-24,49 \%$ y 9.87\%, dejando evidencia que el periodo con índices bajos fue en el 2017.

Maldonado (2018) en su investigación sobre el control de inventarios y su influencia en la rentabilidad de la ferretería Grupo Empresarial Moreno S.A. cuyo nivel del estudio fue causal y enfoque cuantitativo, concluye que el control de existencias no es eficiente, visto que los beneficios económicos fueron mínimos, además que fue comprobado con la $\mathrm{R}$ de Pearson, que los indicadores del control de inventarios influyen moderadamente en los indicadores de rentabilidad de (0.707). Así también los indicadores de rotación en días demostraron una incidencia directa en la rentabilidad, puesto que se obtuvo un valor $\mathrm{p}=0.04$, y un coeficiente positivo moderado de 0.349 .

\subsection{Control de inventario}

Asimismo, define que el control de inventario o stock es una de las actividades que emplea recursos y un capital para la planificación, programación y control de los productos almacenados, a fin de ofertar un servicio en relación con la demanda, con cualidades de calidad, rapidez y fiabilidad y a menor costo (Olivos et al., 2015 citado por Moreno y Bonilla,2019)

Apunte y Rodríguez (2016) es un conjunto o serie de actividades que controlan el ingreso y salida de los artículos y materiales almacenados, en la espera para una venta posterior o utilización en la ejecución de las actividades económicas.

Cruz (2017) es la verificación y control de los materiales almacenados para su posterior venta al cliente (p.5). En tal sentido las acciones de control conllevan a un seguimiento y monitoreo continuo 
donde su eficacia limitará perdidas por deterioro, extravíos, mermas o desmedros. Ferrer y Ferrer (2019) para el control del inventario es importante considerar la aplicación de la NIC 2, que tiene como objetivo principal establecer el tratamiento contable de las existencias o bienes realizables.

Por otra parte, el control de los inventarios tiene como beneficio de no generar mayores costos por el seguimiento en las operaciones de inventario, pues ello permite tener conocimiento del valor inicial del inventario, compras, devoluciones y rebajas, así también de la cantidad de los productos que ingresan y salen de la empresa, y los existente en los almacenes. Por lo tanto, el desarrollo continuo de acciones de control, son primordiales en las empresas, aun mas cuanto estas cuentan con productos perecibles, pues la inasistencia de acciones de control, incurre a mayores pérdidas y gastos Asimismo, el control de inventario permite una eficiente planificación de los productos ya que proporciona herramientas para estimar a los proveedores en relación a los cumplimiento, precio y calidad de sus productos, conocer el promedio de rotación de los productos a fin de que las empresas tomen decisiones prudenciales en cuanto a la compra y almacenamiento de sus productos (Panchi et al., 2017).

En la investigación para evaluación de la variable "control de inventario" se ha considerado como dimensión al índice de rotación de existencias

Cruz (2017) es un valor relativo y elemental que servirá en la empresa para el control del inventario ya que mide la relación que hay entre las existencias que se encuentra en el almacén y su salida en cuanto a días y veces.

En cuanto a los indicadores del control de inventario se ha considerado a la rotación del inventario y Plazo medio de existencias. Escudero (2019) la rotación de inventario es el número de veces que durante un periodo de tiempo (año, trimestre, mes y etc), se renuevan las existencias y recuperamos la inversión del capital invertido" (p.152). En tal sentido, cuando una empresa adquiere materiales o suministros es importante que se preocupe en vender sus productos en el menor tiempo posible, puesto que forma parte de la inversión, que no se recupera hasta la comercialización del producto, Cuya fórmula es la siguiente: Aprovisionamiento/Inventario

En cuanto, al indicador Plazo medio de existencias, Meana (2017) mide generalmente el número de días que permite cubrir las existencias" (p.72). Esto quiere decir, que el control del inventario puede ser evaluado por índices que demuestren la rotación en días. Esto proporciona el stock de un producto en un momento dado. Cruz (2017) es el tiempo que dispone la empresa de existencias, teniendo en cuenta el consumo registrado de las mismas (p.132), es un dato muy importante puesto que manifiesta el número de días que el inventario se han renovado en el almacén durante un periodo establecido que normalmente es un año, siendo muy importante para la empresa en el control de la adquisición de los productos. Inventarios/Aprovisionamiento*365

\subsection{Rentabilidad}

Por lo que refiere a la variable rentabilidad, es importante su evaluación dentro de las organizaciones, por cuanto expresa la situación económica de las mismas, previendo la disponibilidad de efectivo de largo plazo. En tal sentido, conocer su origen es significativo, en tanto, Contreras y Díaz (2015) la situación financiera es la composición de recursos que sirven para el financiamiento propio de la empresa, siendo clasificada por activos, pasivos y patrimonio.

Corona et al. (2014) la rentabilidad es la capacidad que presenta la empresa en generar utilidades, siendo estos medibles en largo periodos, la misma que evidencia la administración efectiva de los recursos que conforman el activo, pasivo y patrimonio de las empresas, y garantiza a los empresarios la inversión sostenible que produce la empresa.

Una de las maneras de evaluar la rentabilidad es a través de los rendimientos de la empresa, en relación con la ventas, activos y capital, es decir la aplicación de porcentuales que demuestran 
eficazmente las utilidades en las ventas activo y de capital. Para Corona et al. (2014) existen dos rentabilidades más importantes entre ellas la rentabilidad económica y financiera.

Por lo que refiere a la rentabilidad económica, Carballo (2013) mide el rendimiento contable generado por la inversión sin considerar la estructura financiera de la organización (p.50). Muñoz (2019) es el rendimiento que obtiene los activos de la misma, independientemente de cómo estén financiados" (p.117). Esta relación, se puede demostrar o expresar con el desempeño de las inversiones o de los activos de la empresa, si la relación de estas es superior la empresa obtendrá mayores beneficios. Dentro de la rentabilidad económica, se evalúa las siguientes ratios:

En tanto, Corona et al. (2014) es el coeficiente en cuyo numerado se visualiza el beneficio formado por los activos" (p.99). En tal sentido demuestra los beneficios obtenidos por parte de los activos, por consiguiente, se cree, que cuanto mayor sean los activos, mayores ganancias disfrutará la empresa, siempre y cuando estás sean administradas de manera eficiente y continuamente. Los valores óptimos que presenta este ratio son superiores o igual a una unidad, es decir 1. Cuya fórmula es la siguiente: (Utilidad neta) /(Total activos)

En cuanto a la rentabilidad financiera, Corona et al. (2014) es entendida como la relación entre los resultados del ejercicio y la inversión efectuada por los propietarios o socios, que se considera semejante al capital. (p.103). En tal sentido demuestra el comportamiento de los recursos propios de la empresa, sin considerar las utilidades generadas por las actividades económicas desarrolladas por la empresa.

En ello se evalúa la rentabilidad neta, Pacheco (2013) este indicador mide la utilidad por cada sol de ventas, cuyo calculo parte de la división de la utilidad neta entre las ventas (p 157). Es importante en el cálculo de la rentabilidad, por cuanto estima uno de los factores elementales dentro de un negocio que es la cantidad de ventas, para toda organización es importante tener una idea del comportamiento de las ventas y como estas contribuyen en el crecimiento de la economía o la generación de utilidades. Corona et al. (2014) es el coeficiente que demuestra el rendimiento de las ventas y su relación con las utilidades, cuyos valores deben situarse en una unidad (1) o mayores a este (p.102), cuya fórmula es: (Utilidad neta)/(Total ventas)

Otro indicador es la rentabilidad sobre el capital, Corona et al. (2014) que mide el resultado después de impuestos y el capital que los engloba, cuyos valores óptimos son mayores o iguales a una unidad (1)", y se obtiene dividiendo los beneficios y el capital. Es importante mencionar, que si la empresa cuenta con recursos significativos y estos son participes de las actividades económicas, la empresa obtendrá mayores ganancias, es por ello que deben ser administrados de manera eficaz y control que demanda la misma, cuya fórmula es: (Utilidad neta)/Capital

\section{PROCEDIMIENTOS METODOLÓGICOS}

\subsection{Tipo y diseño}

La investigación fue de enfoque cuantitativo de tipo aplicada. En tal sentido, Hernández et al. (2014) sostienen que se fundamenta en la evaluación de las características de las variables, lo cual supone proceder de un marco conceptual pertinente del problema evaluado, un conjunto de premisas que detallen las relaciones de las variables analizadas de manera deductiva. La finalidad es incrementar conocimiento en relación con las variables de estudio, a fin de darle una utilidad en la solución de problemas que puedan presentarse en el futuro. 
Conforme la metodología del estudio, el diseño es No experimental, por cuanto se ha observado y analizado los resultados de acuerdo como estos se presentan, limitando el control de estos (Hernández et al., 2014)

\subsection{Población y muestra}

En la población se ha considerado, 48 estados financieros de la empresa "El mundo de los Repuestos JR E.I.R.L", cuyos elementos a evaluar estuvo determinada por el acervo documentario que expresa el rendimiento económico y el control del inventario. En los criterios de exclusión, se consideraron aquellos documentos de años que no comprenden la investigación y aquellos donde no se visualiza la rotación del inventario.

La muestra de la investigación ha sido determinada por 48 estados financieros (24 estados de situación financiera y 24 estados de resultados mensuales).

El tipo de muestreo que se empleó fue el no probabilístico intencional, donde le otorga al autor de la investigación la libertad de escoger a los elementos que cree conveniente para dar solución a los problemas identificados. Por su parte, Arbaiza (2019) el muestreo es una técnica que sirve como un medio eficaz para la determinación de unidades y elementos que participarán en la investigación como población para la solución de los objetivos propuestos en la investigación.

\subsection{Técnica e instrumento}

Se ha determinado como técnica el análisis documental que tiene como función el análisis de los 48 estados financieros. Según, Arbaiza (2019) es la habilidad de recolectar información concerniente una realidad estimada, siendo determinada por el investigador como un medio idóneo para la obtención y clasificación de información que le permitirá captar resultados asertivos y cercanos a la realidad.

En la investigación se ha empleado como instrumento la guía de análisis documental, que ha facilitado la recolección de datos relacionados a los indicadores o ratios expuestos en las teorías que detallan el control de inventario y rentabilidad. Para la aplicación del instrumento, será necesario contar con los 48 estados financieros mensuales de los periodos 2018 - 2019.

\subsection{Procedimiento}

Para la recolección de datos ha sido necesario emitir una carta de autorización para disponer de los estados financieros mensuales de los periodos 2018 al 2019, tras su aceptación, se ha efectuado un análisis aplicando los ratios determinados en la investigación, los mismos que fueron procesados y analizados en hojas de Excel, así también a través del empleo de IBM SPSS25 que ha facilitado su tabulación y representación gráfica, para posteriormente ser interpretados de manera eficiente.

De acuerdo con la metodología de la investigación, se ha empleado como análisis de datos la estadística descriptiva, según Maldonado (2018), este método consiste esencialmente en el estudio de las distribuciones de las variables, permite captar un perfil de los elementos o muestra en cuanto a su caracterización y cualidades. Para representar las variables cuantitativas se pueden utilizar proporciones de tendencia central como (media, mediana y moda) y medidas de dispersión (rango, desviación estándar, varianza), en relación con el nivel de medición o la escala de las variables. En ese sentido para representar correctamente los resultados en el informe, en primera instancia se debe describir las puntuaciones obtenidas por los elementos que integra la muestra, diseñando una distribución de frecuencias.

Así también se ha empleado la estadística inferencial, como primer paso, es importante determinar un tipo de prueba estadística, que en el caso de la investigación se ha empleado la Prueba de Shapiro-Wilk, que es utilizados para analizar la normalidad de las variables, cuyos elementos son 
menores a 50 sujetos, como segundo paso, es definir el nivel se significancia, posterior obtener los datos de la muestra, estimar la desviación estándar, como paso importante es tomar la decisión estadística, a través del coeficiente de correlación de Pearson y finamente emitir una concusión que se ajuste a los objetivos de la investigación.

Durante el desarrollo de la investigación, se ha empleado principios éticos y morales, a través de reglas y normas de conducta, fundamentados desde aspectos y principios de beneficencias, justicia y respeto hacia los involucrados, donde la recolección de información confidencial nace desde el consentimiento brindado por el representante de la institución.

\section{RESULTADOS Y DISCUSIÓN}

\subsection{Resultados Descriptivo}

Tabla 1

Ratios financieros del control de inventarios y rentabilidad de los años 2018 y 2019

\begin{tabular}{lrrrrr}
\hline \multicolumn{1}{c}{ Meses } & \multicolumn{2}{c}{ Control de inventario } & \multicolumn{2}{c}{ Rentabilidad } \\
\cline { 2 - 6 } & $\begin{array}{c}\text { Rotación de } \\
\text { Inventario }\end{array}$ & $\begin{array}{c}\text { Plazo Medio de } \\
\text { existencias }\end{array}$ & $\begin{array}{c}\text { Rentabilidad sobre } \\
\text { activos }\end{array}$ & $\begin{array}{c}\text { Rentabilidad } \\
\text { neta }\end{array}$ & $\begin{array}{c}\text { Rentabilidad } \\
\text { sobre capital }\end{array}$ \\
\hline Ene-18 & 3.20 & 9.39 & 0.08 & 0.07 & 0.67 \\
Feb-18 & 5.48 & 5.47 & 0.04 & 0.02 & 0.19 \\
Mar-18 & 1.30 & 23.14 & 0.06 & 0.03 & 0.31 \\
Abr-18 & 4.23 & 7.09 & 0.21 & 0.11 & 1.05 \\
May-18 & 5.57 & 5.38 & 0.10 & 0.05 & 0.50 \\
Jun-18 & 4.02 & 7.46 & 0.14 & 0.11 & 0.79 \\
Jul-18 & 2.46 & 12.19 & 0.11 & 0.07 & 0.59 \\
Ago-18 & 1.88 & 15.94 & 0.11 & 0.06 & 0.61 \\
Set-18 & 1.85 & 16.22 & 0.05 & 0.07 & 0.84 \\
Oct-18 & 4.02 & 7.45 & 0.07 & 0.05 & 0.33 \\
Nov-18 & 1.94 & 15.46 & 0.13 & 0.09 & 0.72 \\
Dic-18 & 1.69 & 17.79 & 0.02 & 0.03 & 0.41 \\
Ene-19 & 2.05 & 14.63 & 0.02 & 0.04 & 1.01 \\
Feb-19 & 3.58 & 8.39 & 0.13 & 0.08 & 0.83 \\
Mar-19 & 1.18 & 25.41 & -0.01 & -0.18 & -0.08 \\
Abr-19 & 1.21 & 24.81 & -0.05 & -0.26 & -0.37 \\
May-19 & 2.49 & 12.03 & 0.03 & 0.04 & 2.18 \\
Jun-19 & 2.11 & 14.21 & 0.04 & 0.11 & 1.61 \\
Jul-19 & 2.98 & 10.08 & 0.25 & 0.12 & 1.74 \\
Ago-19 & 2.57 & 11.66 & 0.07 & 0.07 & 1.17 \\
Set-19 & 5.19 & 5.78 & 0.27 & 0.14 & 1.47 \\
Oct-19 & 4.50 & 6.67 & 0.05 & 0.05 & 0.27 \\
Nov-19 & 2.57 & 11.67 & 0.17 & 0.10 & 0.96 \\
Dic-19 & 1.63 & 18.45 & 0.07 & 0.06 & 0.46 \\
\hline & & & &
\end{tabular}

La tabla 1 muestra resultados de las ratios financieras del control de inventario y rentabilidad en los años 2018 y 2019. En lo que respecta a la rotación de inventario, los índices con mayores niveles se presentaron en los meses de febrero (5.48), mayo (5.57) del 2018 y setiembre (5.19) del 2019, lo que indica que el inventario tuvo menor permanencia en el almacén. Sin embargo, el índice con menor rotación se presentó en el mes de marzo del 2019 (1.18). 
En lo que respecta al índice de plazo medio de existencias, refleja que los meses de marzo (23.14) del 2018; marzo (25.41) y abril (24.81) del 2019, fueron los meses con mayores días de rotación de mercaderías; no obstante, en mayo del 2018 se ha presentado menos días (5.38 días).

Por lo que refiere a la rentabilidad sobre activos, los índices mayores se presentan en los meses de julio (0.25) y setiembre (0.27) del 2019, no obstante, en los meses de marzo y abril, los índices fueron -0.01 y -0.05 respectivamente.

En cuanto a la rentabilidad neta, los índices de los meses de julio y setiembre presentan niveles de 0.12 y 0.14 respectivamente. Por otro lado, los meses de marzo y abril, los índices fueron -0.18 y 0.26 respectivamente.

Finalmente, en la rentabilidad sobre capital la participación del capital fue mayor en los meses de mayo (2.18), junio (1.61) y julio (1.74) del 2019. Así también, los meses de marzo y abril, los índices fueron -0.08 y -0.37 respectivamente.

A continuación, se presenta las tendencias, que fueron desarrollados a través de graficas de dispersión, en el cual se evalúo en la empresa "El mundo de los Repuestos JR E.I.R.L", cuya actividad es la venta, mantenimiento y reparación de motocicletas y sus partes, piezas y accesorios.

\section{Figura 1}

Tendencia del índice de rotación de inventario

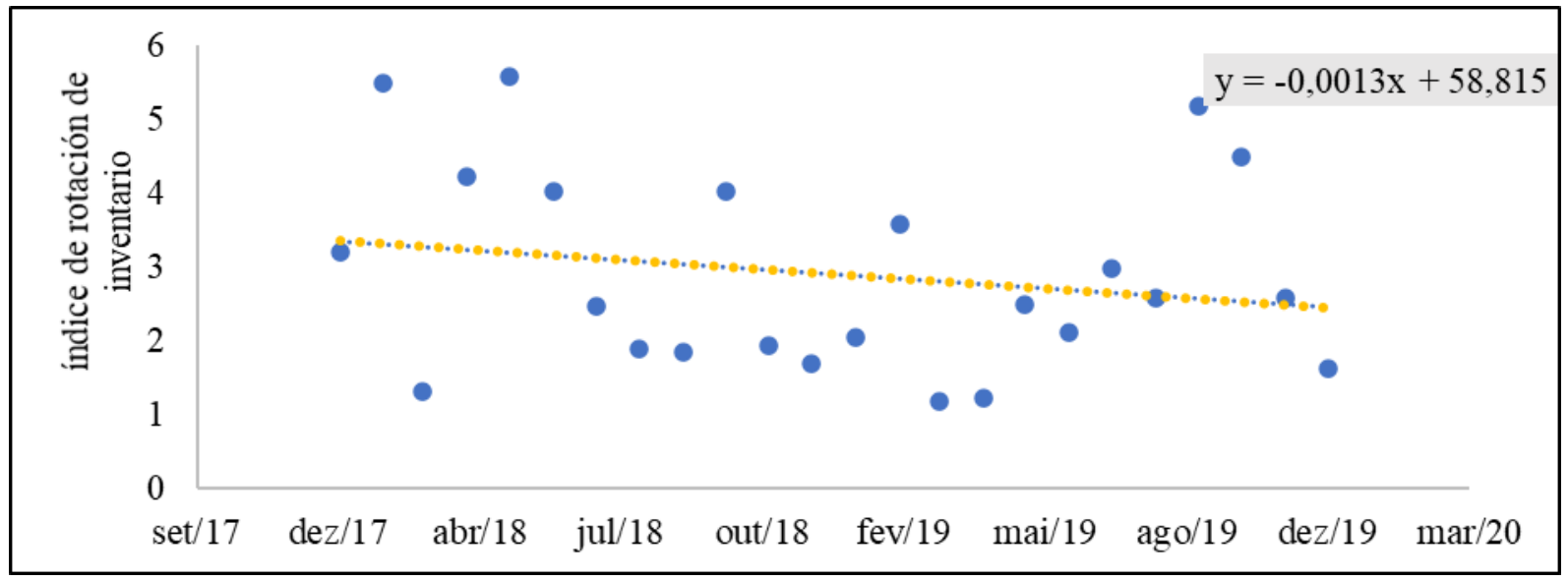

Los datos expuestos en la figura 1, infieren que la línea de tendencia presenta un pendiente = 0.0013, lo que indica que la posición historia del índice de rotación de inventario presenta un comportamiento descendente. Por consiguiente, la tendencia de la variable se debe a los bajos niveles de rotación presentados de manera continua, causantes del sobre stock y el análisis inadecuado de las necesidades, así también del seguimiento y control descontinuo de los productos almacenados y de registros desactualizados, que permiten información errónea y por ende decisiones deficientes. De la misma manera el estudio desarrollado por Asencio y González (2017), concluye que la organización JRC Pharma, que las actividades del control de inventario son deficientes en un $42 \%$ y un $67 \%$ y afirmó que la compañía carece de un control de existencias; asimismo existe deficiente manejo en la adquisición de existencias en un 53\%, por lo cual, ha originado un continuo descenso en los índices de rotación. 
Figura 2

Tendencia del índice de plazo medio de existencias

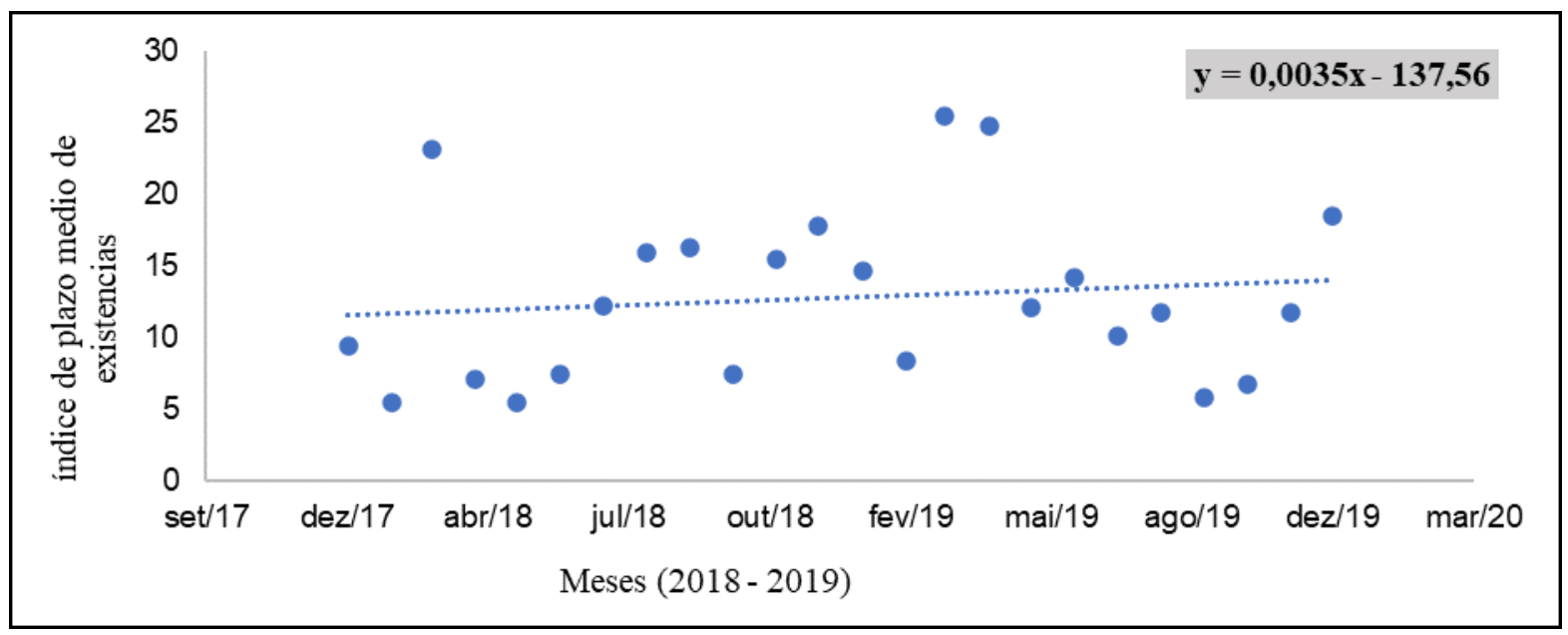

En cuanto a la figura 2, la línea de tendencia, presenta un pendiente $=0.0035>0$, lo que indica que la posición historia del índice de plazo medio de existencias ha experimentado un crecimiento en los periodos estudiados, es decir que el ratio presenta un comportamiento ascendente. Como se observa, los productos almacenados no se mantienen más de un mes en las bodegas, sin embargo, estos deben ser menores, dado que existe productos que presentan deterioro y vencimiento.

\section{Figura 3}

Tendencia del índice de rentabilidad sobre activos

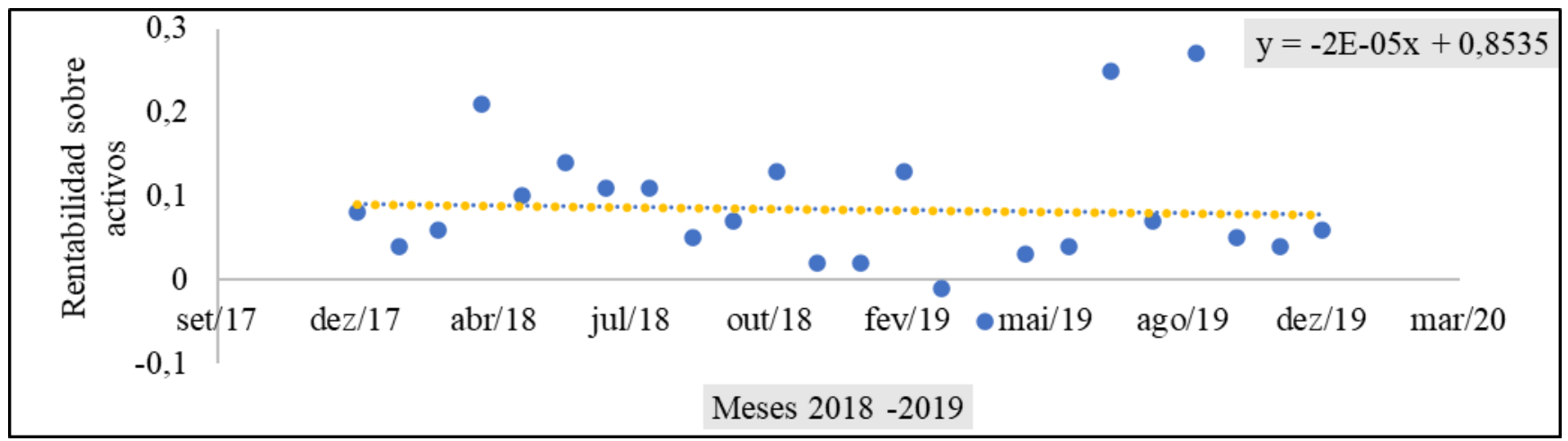

Conforme se evidencia en la figura 3, la línea de tendencia presenta un pendiente $=-2 \mathrm{E}$ lo que indica que la posición historia del índice de rentabilidad sobre activos fue decreciente en los periodos estudiados, es decir que el ratio presenta un comportamiento descendente. La rotación mínima de los productos ha permitido un retorno mínimo del activo circulante, dificultando el cumplimiento de las obligaciones adquiridas por la empresa, así también bajos índices de rentabilidad. De la misma manera el informe efectuado por Jara y Sánchez (2017) concluyó que la organización dejo evidencia de faltantes de mercadería por falta de un control adecuado en el almacén, lo que ha conllevado que exista una incidencia negativa en el rendimiento económico relacionado a los activos de la empresa, es decir, existe dificultades para disponer de recursos para la adquisición de mercadería y el cumplimiento de las obligaciones financieras y con terceros. 
Figura 4

Tendencia del índice de rentabilidad neta

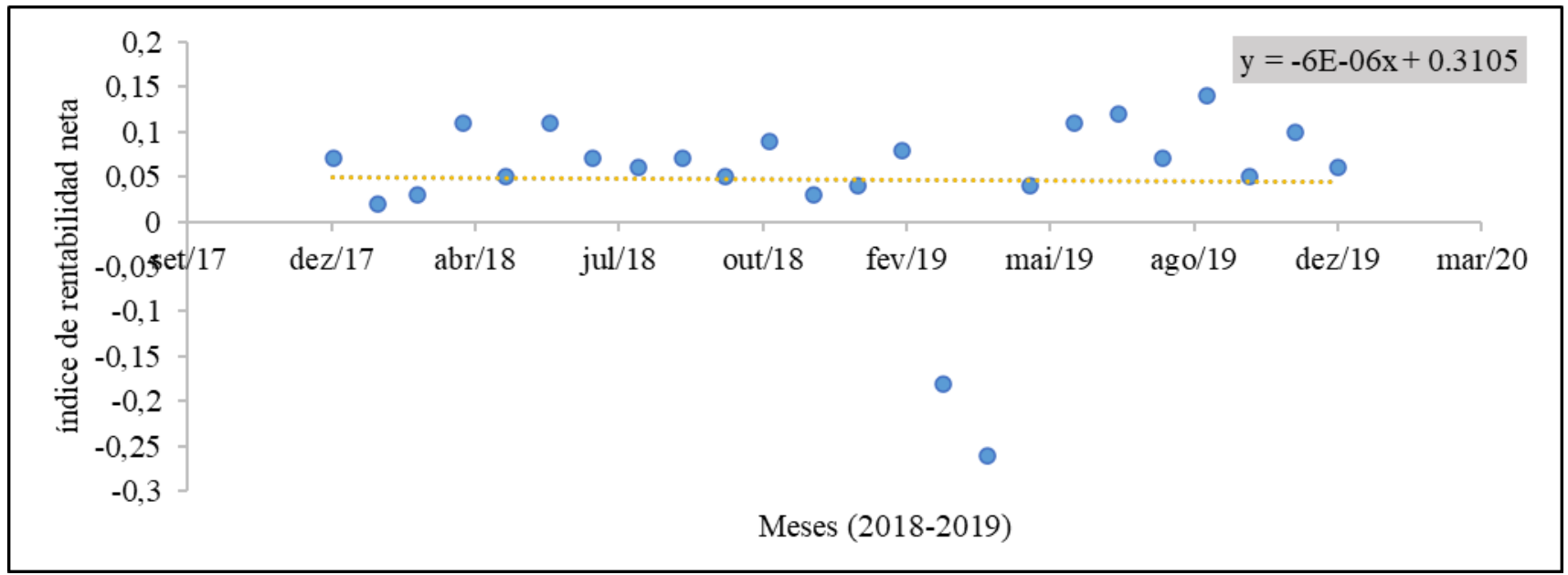

Así también en la figura 4, en lo que respecta al ratio de rentabilidad ha venido presentado una tendencia descendente, por cuanto presenta un pendiente negativo, asimismo se observa que desde febrero hasta mayo, los índices presentan resultados negativos. Además, al existir un bajo nivel de rotación de los productos, permite que las ventas no se desarrollen de la manera que la gerencia espera, como se observa las ventas desde 2018 se ha mantenido, sin embargo, en los últimos periodos ha sufrido un descenso, y por consiguiente la utilidad también se ve afectado, la participación regular de las ventas en las utilidades ha permitido que la rentabilidad neta presente un nivel ascendente.

\section{Figura 5}

Tendencia del indice de rentabilidad sobre capital

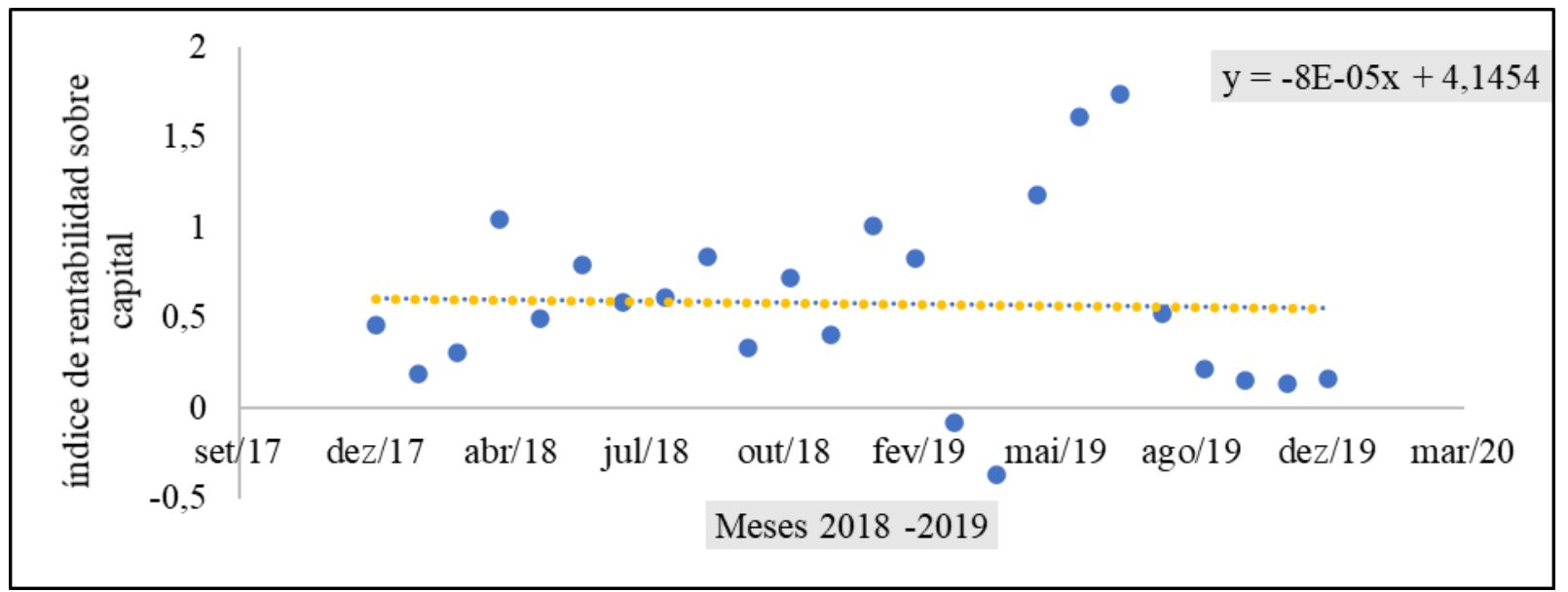

En lo que respecta a la figura 5, la línea de tendencia presenta un pendiente $=-8 \mathrm{E}$, lo que indica que el índice de rentabilidad sobre capital ha experimentado un comportamiento descendente. La rotación del inventario no es un elemento relevante en la rentabilidad de capital, su descenso es ocasionado por otros factores. 


\subsection{Resultados inferenciales}

Tabla 2

Prueba de normalidad

\begin{tabular}{lrrr}
\hline & \multicolumn{2}{c}{ Shapiro-Wilk } & \\
& Estadístico & Gl & \multicolumn{1}{c}{ Sig. } \\
\hline Rotación de inventario & 0.915 & 24 & 0.056 \\
Plazo medio de existencia. & 0.922 & 24 & 0.064 \\
Rentabilidad sobre activos & 0.954 & 24 & 0.329 \\
Rentabilidad neta & 0.671 & 24 & 0.110 \\
Rentabilidad sobre el capital & 0.974 & 24 & 0.758 \\
\hline
\end{tabular}

*. Esto es un límite inferior de la significación verdadera.

a. Corrección de significación de Lilliefors

Se muestra en la tabla 3, la prueba de normalidad de Shapiro-Wilk (para muestras menores a 50 ), donde el $\mathrm{p}$ valor $=>0.05$, en tal sentido los datos proceden de una distribución normal. Por lo tanto, se empleará la prueba paramétrica $r$ de Pearson, con la finalidad de resolver los objetivos que buscan determinar la relación de las variables y dimensiones.

\section{Comprobación de hipótesis}

\section{Hipótesis especifico 01}

H0: No existe relación entre rotación de inventario y rentabilidad sobre activos en la empresa “El mundo de los Repuestos JR E.I.R.L”., Distrito de Manantay, Periodo 2018-2019

H1: Existe relación entre rotación de inventario y rentabilidad sobre activos en la empresa "El mundo de los Repuestos JR E.I.R.L”., Distrito de Manantay, Periodo 2018-2019

\section{Tabla 3}

Relación entre rotación de inventario y rentabilidad sobre activos

\begin{tabular}{llr}
\hline & & \multicolumn{2}{c}{$\begin{array}{c}\text { Rentabilidad sobre } \\
\text { activos }\end{array}$} \\
\hline \multirow{3}{*}{ Rotación de inventario } & Correlación de Pearson &, $450^{*}$ \\
& Sig. (bilateral) & 0.027 \\
& $\mathrm{~N}$ & 24 \\
\hline
\end{tabular}

*. La correlación es significativa en el nivel 0,05 (bilateral).

En la tabla 3 se muestra un p valor $=0.027<0,05$, que prueba que existe relación entre la rotación de inventario y rentabilidad sobre activos. Asimismo, se obtuvo un coeficiente de correlación positiva moderada de 0,450; por lo tanto, se rechaza la hipótesis nula y se acepta la hipótesis que señala "Existe relación entre rotación de inventario y rentabilidad sobre activos en la empresa "El mundo de los Repuestos JR E.I.R.L”., Distrito de Manantay, Periodo 2018-2019”. Asimismo, la investigación de Quinde y Ramos (2018) se relacionan a los resultados obtenidos en el estudio, dado que concluye que la rotación de inventario incide de forma positiva en la rentabilidad, con un p valor $=0.00<0.05$, y con un indicador de correlación de Pearson de 0.781, por cuanto tiene un proceso de venta favorable, y presenta una rotación mensual de 3.94, que ha permitido mayores ganancias. 


\section{Hipótesis especifico 02}

H0: No existe relación entre rotación de inventario y rentabilidad neta en la empresa "El mundo de los Repuestos JR E.I.R.L”., Distrito de Manantay, Periodo 2018-2019.

H1: Existe relación entre rotación de inventario y rentabilidad neta en la empresa "El mundo de los Repuestos JR E.I.R.L”., Distrito de Manantay, Periodo 2018-2019.

\section{Tabla 4}

Relación entre rotación de inventario y rentabilidad neta

\section{Rentabilidad neta}

\begin{tabular}{llr}
\hline & Correlación de Pearson &, $407^{*}$ \\
Rotación de inventario & Sig. (bilateral) & 0.048 \\
& $\mathrm{~N}$ & 24 \\
\hline
\end{tabular}

*. La correlación es significativa en el nivel 0,05 (bilateral).

En la tabla 4 se muestra un p valor $=0,048<0,05$, que prueban que existe relación entre rotación de inventario y rentabilidad neta. Además, el coeficiente de correlación es 0,407, lo que evidencia que existe relación positiva moderada. Es por ello que se rechaza la hipótesis nula y se acepta la hipótesis que indica "Existe relación entre la rotación de inventario y rentabilidad neta en la empresa “El mundo de los Repuestos JR E.I.R.L”., Distrito de Manantay, Periodo 2018-2019. Los resultados de la investigación guardan relación con los obtenidos por Terán (2017), quien concluye que existe un efecto positivo entre el control de inventario y la rentabilidad, visto que el nivel de significancia fue $=$ 0,01, cuya correlación de Rho Spearman es 0.745 , de tal modo se aceptó la hipótesis alterna y se rechazó la hipótesis nula. En tal sentido, el movimiento continuo de las existencias contribuirá que las ventas sean mayores y la empresa tenga la facilidad de pagar y adquirir nuevas obligaciones, para el bienestar de la misma.

\section{Hipótesis especifico 03}

H0: No existe relación entre rotación de inventario y rentabilidad sobre el capital en la empresa “El mundo de los Repuestos JR E.I.R.L”., Distrito de Manantay, Periodo 2018-2019.

H1: Existe relación entre rotación de inventario y rentabilidad sobre el capital en la empresa "El mundo de los Repuestos JR E.I.R.L”., Distrito de Manantay, Periodo 2018-2019.

\section{Tabla 5}

Relación entre rotación de inventario y rentabilidad sobre el capital

\begin{tabular}{llr}
\hline & & Rentabilidad sobre el capital \\
\hline \multirow{3}{*}{ Rotación de inventario } & Correlación de Pearson & 0.097 \\
& Sig. (bilateral) & 0.652 \\
& $\mathrm{~N}$ & 24 \\
\hline
\end{tabular}

*. La correlación es significativa en el nivel 0,05 (bilateral).

En la tabla 5 se muestra un p valor $=0,652>0,05$, que prueban que no existe relación entre rotación de inventario y rentabilidad sobre el capital. Por lo tanto, se rechaza la hipótesis alterna y se acepta la hipótesis que señala "No existe relación entre la rotación de inventario y la rentabilidad sobre 
el capital en la empresa "El mundo de los Repuestos JR E.I.R.L”., Distrito de Manantay, Periodo 20182019”. La investigación de Asencio y González (2017), muestra resultados semejantes, ya que concluyo que el índice de rentabilidad del capital no muestra dependencia del control de inventario, visto que el $\mathrm{p}$ valor $=0.091>0.05$. En tal sentido, En el estudio, los resultados descriptivos evidencian que los índices de rentabilidad tuvieron movimientos volubles, donde los índices con mayor significancia fueron en los meses de mayo (2.18), junio (1.61) y julio (1.74) del 2019.

\section{Hipótesis especifico 04}

H0: No existe relación entre plazo medio de existencias y rentabilidad sobre activos en la empresa "El mundo de los Repuestos JR E.I.R.L”., Distrito de Manantay, Periodo 2018-2019.

H1: Existe relación entre plazo medio de existencias y rentabilidad sobre activos en la empresa “El mundo de los Repuestos JR E.I.R.L”., Distrito de Manantay, Periodo 2018-2019.

\section{Tabla 6}

Relación entre plazo medio de existencia y rentabilidad sobre activo

\begin{tabular}{llr}
\hline & & Rentabilidad sobre activos \\
\hline \multirow{3}{*}{ Plazo medio de existencia. } & Correlación de Pearson &,$- 556^{* *}$ \\
& Sig. (bilateral) & 0.005 \\
& $\mathrm{~N}$ & 24 \\
\hline **.
\end{tabular}

**. La correlación es significativa en el nivel 0,01 (bilateral).

En la tabla 6 se muestra un p valor $=0,005<0,05$, prueba que existe relación entre el plazo medio de existencia y rentabilidad sobre activo. En lo que respecta al coeficiente de correlación, muestra que existe una relación negativa moderada de -0.556 , por lo tanto, se rechaza la hipótesis nula y se acepta la hipótesis que indica "Existe relación entre plazo medio de existencias y rentabilidad sobre activos en la empresa "El mundo de los Repuestos JR E.I.R.L"., Distrito de Manantay, Periodo 2018-2019”. Los resultados presentados por Maldonado (2018) guardan relación con los obtenidos en el estudio, visto que concluye que los indicadores de rotación en días demostraron una incidencia directa en la rentabilidad, cuyo $\mathrm{p}$ valor $=0.04<0.05$, y un coeficiente positivo moderado de 0.349 . Así también demostró que el control de existencias no es eficiente, visto que los beneficios económicos fueron mínimos, además que fue comprobado con la $\mathrm{R}$ de Pearson, que los indicadores del control de inventarios influyen moderadamente en los indicadores de rentabilidad de (0.707).

\section{Hipótesis especifico 05}

H0: No existe relación entre plazo medio de existencias y rentabilidad neta en la empresa "El mundo de los Repuestos JR E.I.R.L”., Distrito de Manantay, Periodo 2018-2019;

H1: Existe relación entre plazo medio de existencias y rentabilidad neta en la empresa "El mundo de los Repuestos JR E.I.R.L”., Distrito de Manantay, Periodo 2018-2019. 
Tabla 7

Relación entre plazo medio de existencia y rentabilidad neta

\begin{tabular}{llr}
\hline & & $\begin{array}{c}\text { Rentabilidad } \\
\text { neta }\end{array}$ \\
\hline & Correlación de Pearson &,$- 665^{* *}$ \\
Plazo medio de existencia. & Sig. (bilateral) & 0.000 \\
& $\mathrm{~N}$ & 24 \\
\hline
\end{tabular}

**. La correlación es significativa en el nivel 0,01 (bilateral).

En la tabla 7 se muestra un p valor $=0,000<0,05$, que prueban que existe relación entre el plazo medio de existencia y rentabilidad neta. Asimismo. existe un coeficiente de correlación negativa moderada de -0.665. Por lo tanto, se rechaza la hipótesis nula y se acepta la hipótesis que infiere "Existe relación entre plazo medio de existencias y rentabilidad neta en la empresa "El mundo de los Repuestos JR E.I.R.L”., Distrito de Manantay, Periodo 2018-2019”.Los resultados se asemejan a los alcanzados por López y Galarreta (2018), quienes desde un método descriptivo concluyen que los días que permanece el inventario dentro de los almacenes no son mayores a 12 días por lo que ha tenido un efecto positivo en las ventas S/ 56,521.00 y diversificación de los productos en el periodo 2019. Así mismo en el estudio, se conoció que en los meses de marzo (23.14) del 2018; marzo (25.41) y abril (24.81) del 2019 el inventario tuvo mayor permanencia en los almacenes, no obstante, en mayo del 2018, se ha presentado menos días (5.38 días). Así también, ha presentado una tendencia ascendente de 0.0421 .

\section{Hipótesis especifico 06}

H0: No existe relación entre plazo medio de existencias y rentabilidad sobre el capital en la empresa "El mundo de los Repuestos JR E.I.R.L”., Distrito de Manantay, Periodo 2018-2019.

H1: Existe relación entre plazo medio de existencias y rentabilidad sobre el capital en la empresa “El mundo de los Repuestos JR E.I.R.L”., Distrito de Manantay, Periodo 2018-2019.

\section{Tabla 8}

Relación entre plazo medio de existencia y rentabilidad sobre el capital

\section{Rentabilidad sobre el capital}

Correlación de Pearson

Sig. (bilateral)

$\mathrm{N}$
$-0.357$

0.008

24

**. La correlación es significativa en el nivel 0,01 (bilateral).

En la tabla 8 , se observa un p valor $=0,008<0,05$, en tal sentido, la relación entre el plazo medio de existencia y rentabilidad sobre el capital es estadísticamente significativa, es decir hay relación entre las mismas. En lo que respecta al coeficiente de correlación, muestra que existe una relación negativa baja de -0.357 , por lo tanto, se rechaza la hipótesis nula y se acepta la hipótesis que indica "Existe relación entre plazo medio de existencias y rentabilidad sobre el capital en la empresa “El mundo de los Repuestos JR E.I.R.L”., Distrito de Manantay, Periodo 2018-2019”. Por el contrario, Asencio y González (2017), sostiene que no existe relación entre los indicadores del control de 
inventario y rentabilidad del capital, dado que la rotación de las existencias no tiene repercusión en el capital de la empresa, no obstante, son otros factores que inciden en la rentabilidad del capital.

\section{CONCLUSIÓN}

Tras un análisis estadístico se ha llegado a concluir que existe relación significativa y directa entre control de inventarios y rentabilidad en la empresa "El mundo de los Repuestos JR E.I.R.L.", Distrito de Manantay, periodo 2018-2019. Sin embargo, se determinó que no existe relación entre rotación de inventario y rentabilidad sobre el capital, entonces mientras menor sea la rotación del inventario no afecta de ninguna manera a la rentabilidad sobre el capital.

En lo que respecta a las limitaciones presentadas en la investigación, la muestra no fue suficientemente representativa para lograr recolectar más información, es decir, es necesario considerar la percepción de los jefes de área, para identificar de manera clara las deficiencias en relación con el control de inventario mediante la aplicación de una guía entrevista.

A raíz de los resultados, como agenda futura de investigación se presentan las siguientes interrogantes: ¿Existen diferencias significativas en el control de inventarios en las empresas ferreteras de la Región de San Martín?, ¿Cuáles son las estrategias de mayor eficiencia en el control de inventario en las empresas sanmartinenses?, y ¿Existirá diferencia significativa entre los niveles de rotación de inventarios antes y durante la COVID-19 en las empresas ferreteras de San Martín?

\section{REFERENCIAS}

Ángulo, R. (2019). Control interno y gestión de inventarios de la empresa constructora Peter Contratistas S.R. Ltda. Revista de la universidad de Huanúco, 5(2), 129-137. Obtenido de http://revistas.unheval.edu.pe/index.php/gacien

Apunte, R., \& Rodriguez, R. (2016). Diseño y aplicación de sistema de gestión en Inventarios en empresa ecuatoriana. Ciencias Holguín,, 22(3), 1-14. Obtenido de https://www.redalyc.org/pdf/1815/181546432006.pdf

Arbaiza, L. (2019). Como elaborar una tesis de grado (1 ed.). Colombia: Esan Ediciones.

Asensio, L., \& Gónzales, E. (2017). El inventario como determinante en la rentabilidad de las distribuidoras farmacéuticas. Revista de Ciencias de la Administración y Economía, 7(13), 1-18. Obtenido de https://www.redalyc.org/jatsRepo/5045/504551272009/504551272009.pdf

Carballo, J. (2013). El diagnostico económico -financiero de la empresa. (1 ed.). Madrid: ESIC Editorial.

Contreras, N., \& Diáz, E. (2015). Estructura financiera y rentabilidad: origen, teorías y definiciones. Revista Valor contable, 2(1), 35-44. Obtenido de https://revistas.upeu.edu.pe/index.php/ri_vc/article/view/824

Corona, E., Bejarano, V., \& Gónzalez, J. (2014). Análisis de estados finaniceros individuales y consolidados. (1 ed.). Madrid: UNED.

Cruz, A. (2017). Gestión de inventario COML0210 (1 ed.). España: IC Editorial.

Escudero, J. (2019). Logística de almacenamiento. (2 ed.). España: Ediciones Paraninfo.

Hernández, R., Fernández, C., \& Baptista, P. (2014). Metodología de la investigación (6 ed.). México: Editorial Mc Griwil.

Jara, S., \& Sanchez, D. (2017). Análisis para la mejora en el manejo de inventarios de una comercializadora. Revista de Ingeniería Industrial., 1(1), 1-18. Obtenido de https://n9.cl/b6vf

López, B., \& Galarreta, G. (2018). Gestión de inventarios para reducir los costos del almacén de Manpower Perú E.I.R.L. Revista de investigación cientifica Ingnofis, 4(1), 15-28. Obtenido de http://revistas.ucv.edu.pe/index.php/INGnosis/article/view/2058/1743 
Maldonado, J. (2018). Metodología de la investigación Social. Cuantitativo, sociocrítico, cualitativo, complementario (1 ed.). Colombia: Ediciones de la U.

Maldonado, M. (2018). Evaluación del control de inventarios y su influencia en la rentabilidad de la ferretería Grupo Empresarial Moreno S.A.C - Nuevo Chimbote, 2017. Universidad Cesar Vallejo, Chimbote, Perú.

Manosalva, L., Baque, L., \& Peñafiel, G. (2020). Estrategia de control interno para el área de inventarios en la empresa Ferricortez comercializadora de productos ferreteros en el cantón Santo Domingo. Revista Universidad y Sociedad, 12(4). Obtenido de http://scielo.sld.cu/scielo.php?script=sci_arttext\&pid=S2218-36202020000400288

Meana, P. (2017). Gestión de inventario (1 ed.). España: Ediciones Nobel.

Moreno, K., \& Bonilla, D. (2019). Logística y control de stock. Caso de estudio en librerías y papelerías. Revista Venezolana de Gerencia, 24(88). Obtenido de https://www.redalyc.org/jatsRepo/290/29062051020/29062051020.pdf

Pacheco, J. (2013). Contabilidad financiera con Excel. (1 ed.). Lima, Perú: Editorial Macro.

Panchi, V., Armas, I., \& Chasi, B. (2017). Los inventarios y el costo de producción en las empresas industriales del Ecuador. Roca, 13(4). Obtenido de https://dialnet.unirioja.es/servlet/articulo?codigo $=6759713$

Peña, O., \& Silva, R. (2016). Factores incidentes sobre la gestión de sistemas de inventario en organizaciones Venezonalas. Telos, 18(2), 187-207. Obtenido de https://www.redalyc.org/pdf/993/99345727003.pdf

Quinde, C., \& Ramos, T. (2018). Valuación y control del inventario y su efecto en la rentabilidad. Revista Observatorio de la Economía Latinoamericana, 2(12), 1-12. Obtenido de https://www.eumed.net/rev/oel/2018/07/inventariorentabilidad.html

Segura, M. (2019). Operaciones vinculadas y rentabilidad de la empresa A. Hatrodt Perú S.A.C, 2016-2018. Revista de la Universidad Norbert Wiener, 4(3), 74-84. Obtenido de https://doi.org/10.33890/innova.v4.n3.2019.1022

Terán, S. (2017). El control de los inventarios (NIC 2) en la rentabilidad de la empresa PROVEC INDUSTRIAL de la ciudad de Ambato. Universidad Técnica de Ambato, Ambato, Ecuador. Obtenido de https://n9.cl/964h 